\section{Crystal Structure of Rochelle Salt}

We have succeeded in finding approximate positions for all the atoms of Rochelle salt (sodium potassium tartrate tetrahydrate) in the crystal structure. The unit cell has dimensions $11.93 \mathrm{~A} . \times$ $14 \cdot 30 \mathrm{~A} . \times 6 \cdot 17 \mathrm{~A} .(Z=4)$, and the space group is $P 2_{1} 2_{1} 2$. The set of general positions expressing this symmetry is

$x y z ; \bar{x} \bar{y} z \frac{1}{2}+x, \frac{1}{2}-y, \bar{z} ; \frac{1}{2}-x, \frac{1}{2}+y, \bar{z}$, and the co-ordinates of the various atoms are as follows (expressed in sixtieths of the cell edges) :

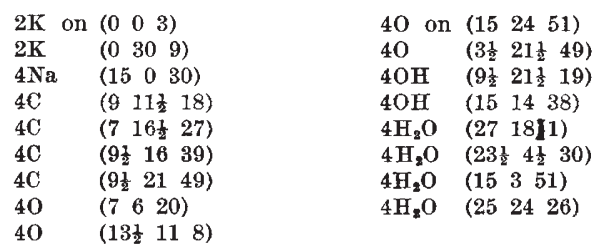

We are not satisfied with the accuracy of these positions; but we have no doubt that our general idea of the structure is correct. It agrees with twodimensional Fourier syntheses projected down the $a$ and $b$ axes (the signs of the $F$ 's being obtained from intensity changes in the differently substituted salts) and also with a three-dimensional Patterson diagram of the ammonium salt, for which all the intensities were observed. All the interatomic distances are reasonable. The water molecules seem to be either of the three- or the four-bonded type. They coordinate the sodium and potassium atoms and assist in bonding these to the tartrate molecule. The tartrate molecule itself has an extended carbon chain with the two identical halves of the molecule lying in two planes inclined at $60^{\circ}$ with one another.

It is hoped to derive more accurate positions and to publish a fuller account of the structure very soon.

Dewar Crystallographic Laboratory,

C. A. Benvers.

W. Hughes.

University of Edinburgh. June 25.

\section{Calculation of Energy Absorbed in Irradiated Tissue}

IN a previous letter ${ }^{1}$, a method was outlined for the volume integration of dosage for $\mathrm{X}$ - and gammaray beams of radiation. The result of the calculations for a beam of X-rays passing through a "water phantom' from a $200 \mathrm{kv}$. Metropolitan-Vickers X-ray tube filtered through $1 \mathrm{~mm}$. copper and $1 \mathrm{~mm}$. aluminium at a focal skin distance of $40 \mathrm{~cm}$. showed the integrated dosage $R$, to be

$R=5.6 \times A r_{0}+\frac{A r_{0}}{0.085}\left(1-e^{-0 \cdot 085(x-5 \cdot 6)}\right)$,

where $A$ is the area of the applicator at the skin surface and $r$ the maximum skin dose for a large applicator $(20 \mathrm{~cm} . \times 15 \mathrm{~cm}$.).

When applied clinically, the above formula allows of comparison of energy absorbed by patients during treatment; but for the computation of absolute energy further calculations are necessary. A method of deducing these absolute values using the ionization method of measurement in small air chambers in the irradiated material has been devised by Gray ${ }^{2}$, who states that... "If secondary beta radiation is being generated in a medium $M$ at a rate $E_{v}$ ergs c.c./sec. then the rate of production of ions in a gaseous medium enclosed in a small cavity in $M$ is $J_{v}=E_{v} / W_{\rho}$. . . . (2), where $W$ is the average energy lost by a beta particle per pair of ions produced and $\rho$ is the ratio of the stopping power of $M$ and the gaseous medium for the particles concerned". Since $R$ is the total integrated dosage in a volume $V$, then $\int_{0}^{V} J_{v} d v=R / t . e$, where $e$ is the charge in E.S.U. on an electron, and $t$ the time in seconds to liberate the charge. The energy absorbed from an X-ray beam passing through a homogeneous medium is therefore

$$
E=\frac{R \times W \times p}{e}
$$

The validity of this equation, however, depends on certain definite conditions ${ }^{2}$. On applying these to the present problem, it is seen to be essential that the material of the absorbing phantom and the dosimeter walls have the same value of $\rho$, and that the ratio of loss of energy of a beta particle producing an ion pair in air and in the medium be known. The results ${ }^{3}$ used in the calculation of integrated dosage were obtained by means of dosimeters the walls of which had approximately the same mean atomic number and electronic density as the water of the phantom; but these walls had been rendered conducting with a film of graphite. Thus the assumption that $\rho$ is the same for dosimeter walls and water is not correct, but for clinical work where homogeneity is non-existent, and the electronic density of tissue only approximates to that of water, the results may be regarded as sufficiently accurate.

The ratio of the number of electrons per c.c. in air at N.T.P. and in water is $0.858 \times 10^{3}$; then the quantity of charge $T$ liberated in tissue as compared with air is given by

$$
T=R \times 0.858 \times 10^{3} \text { E.S.U. }
$$

The energy liberated in tissue is $E=\frac{R \times W \times 0.858 \times 10^{3}}{4.77 \times 10^{-10}}$;

where $W$, the mean energy required to produce an ion pair in air, is $32.5 \mathrm{eV}$. or $5 \cdot 2 \times 10^{-11} \mathrm{ergs}$. (Since this letter was written, it has been shown by Lasnitzki and Lea that the relative ionization in tissue for equal ionization in air for gamma and hard $\mathrm{X}$-rays is 1.12 ; thus the energy absorbed in tissue from the beam considered above is probably 12 per cent greater than the value given.) In water with a mean atomic number of $\mathbf{3} \cdot \mathbf{3 3}, W$ (water) becomes $1 \cdot 04 \times 5 \cdot 2 \times 10^{-11}$ ergs. The expression, therefore, for the total absorption of energy in tissue during irradiation is

$$
E=\frac{R \times 1.04 \times 5 \cdot 2 \times 10^{-11} \times 0.858 \times 10^{3}}{4 \cdot 77 \times 10^{-10}} \text { ergs ; }
$$

or $E=97 R$ ergs or $2.32 \times 10^{-6} R$ calories. This value is necessarily approximate for the reasons stated above, and for absorption in bone modified values of $p$ and $W$ have to be introduced.

A more detailed discussion of the above is to be given at a later date.

Physics Laboratory,

Frant Happey.

Radium Centre,

Royal Infirmary, Sheffield.

1 Happey, F., NATdRe, 145, 668 (1940).

${ }^{2}$ Gray, I. H., Proc. Roy. Soc., A, 156, 578 (1936).

${ }^{3}$ Parker and Honeyburne, Brit. J. Rad., 8, 684 (1935).

- Lasnitzky and Lea, Brit. J. Rad., 13, 149 (1940), 